\title{
A pulmonary artery false aneurysm after right middle lobectomy: a
} case report

\author{
Hossam Shaaban*1, Hemant Sharma ${ }^{2}$, Jagan Rao $^{1}$ and Stephen Clark ${ }^{1}$
}

\author{
Address: ${ }^{1}$ Cardiothoracic Centre, Freeman Hospital, Newcastle upon Tyne, UK and ${ }^{2}$ Department of Vascular Surgery, Frenchay Hospital, Bristol, \\ UK \\ Email: Hossam Shaaban* - hossam24973@yahoo.com; Hemant Sharma - hemantz1001@gmail.com; \\ Jagan Rao - jagan.rao@nuth.northy.nhs.uk; Stephen Clark - stephen.clark@nuth.northy.nhs.uk \\ * Corresponding author
}

Published: 25 August 2007

Journal of Medical Case Reports 2007, I:70 doi:10.1 186/1752-1947-1-70
Received: 18 December 2006

Accepted: 25 August 2007

This article is available from: http://www.jmedicalcasereports.com/content/l/1/70

(c) 2007 Shaaban et al; licensee BioMed Central Ltd.

This is an Open Access article distributed under the terms of the Creative Commons Attribution License (http://creativecommons.org/licenses/by/2.0), which permits unrestricted use, distribution, and reproduction in any medium, provided the original work is properly cited.

\begin{abstract}
Pulmonary artery false aneurysm is a rare condition, reported to complicate interventional procedures. We report a case of a false aneurysm of the interlobar pulmonary artery following a right middle lobectomy for lung cancer. This is probably the first reported case.
\end{abstract}

\section{Background}

Pulmonary artery false aneurysm is a rare condition, more common in females and with advancing age. It is related to an increase in pulmonary artery pressure [1] and has been described as a complication of vascular interventional procedures [2]. However, pulmonary artery pseudoaneurysm as a postoperative complication of pulmonary resection is unreported. We report a case of a false aneurysm of the interlobar pulmonary artery following a right middle lobectomy.

\section{Case presentation}

A 69-year-old gentleman underwent a right middle lobectomy for a Stage 1A squamous cell carcinoma of his right middle lobe. The operation involved stapling the pulmonary veins using TX30V stapler and double ligation of the pulmonary artery branches. The bronchus was stapled using a TA30 stapler and the minor fissure was completed with a TCT75 linear cutting stapler. The early post-operative course was unremarkable. The patient however was readmitted five weeks postoperatively with pyrexia and an episode of haemoptysis. Chest X-ray showed haziness in the right mid-zone and the patient was treated for a presumed chest infection. He improved and was discharged.
A week later, he was readmitted with continuing haemoptysis, swinging pyrexia and increasing shortness of breath at rest. Bronchoscopy revealed granulation tissue near the middle lobe bronchial stump, which bled profusely on biopsy. Blood appeared to be venous in origin. A clot in the lower lobe bronchus was found together with an inflamed and narrowed lower lobe orifice. CT scan showed a false aneurysm of the right descending interlobar pulmonary artery and complete lower lobe collapse and consolidation (Fig 1). The diagnosis of a bronchovascular fistula complicating a false aneurysm was made and the patient was re-explored with a view to completion right lower lobectomy.

At the second thoracotomy, there were dense and vascular adhesions throughout the pleural space, which was completely obliterated. Mobilisation of the lung was extremely difficult. The entire lung was consolidated and solid. The pulmonary veins were exceedingly friable and attempt to encircle them caused the vessels to tear and result in massive bleeding. In order to maintain vascular control, a pneumonectomy was considered the safest option under the circumstances. Postoperatively, the 


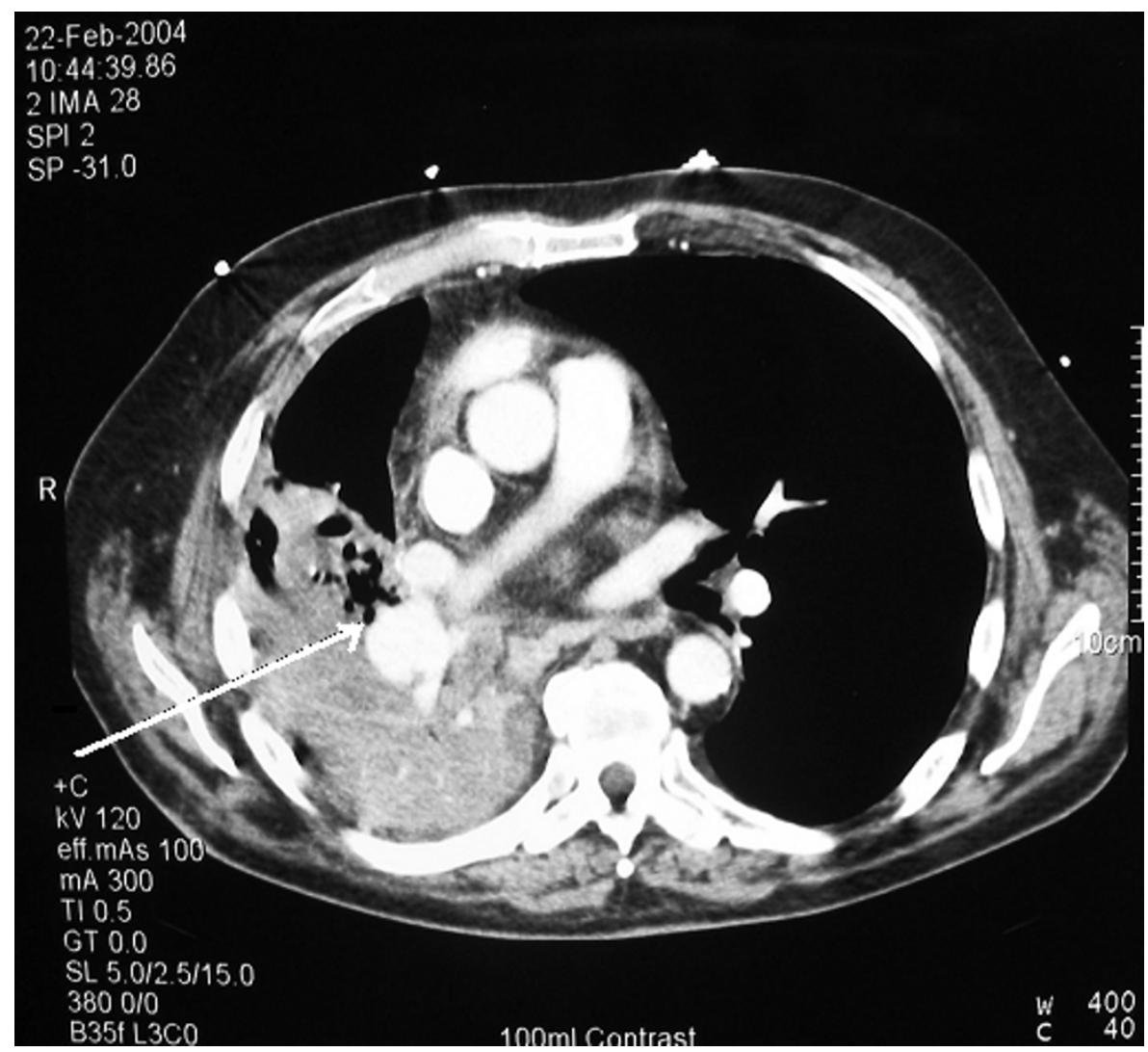

Figure I

False aneurysm of the right descending interlobar artery (arrow). Note consolidation of the lung parenchyma.

patient recovery was unremarkable and was discharged home on the eighth postoperative day.

Macroscopic examination of the bilobectomy specimen showed a grossly consolidated lung, distorted by adhesions and pleural thickening. On incision of the pulmonary artery, atheromatous change was noted in the proximal branching of the artery and the false aneurysm cavity was seen communicating with the bronchus intermedius (Fig 2). Microscopic examination of the specimen showed a quite marked atheroma of the pulmonary artery and a false aneurysm with adjacent abscess like inflammatory mass. Lung parenchyma showed an organising pneumonia, oedema and intra-alveolar fibrin.

Examination of the bronchial margin and the lung parenchyma showed no residual carcinoma.

\section{Discussion}

False aneurysms result from rupture of all three structural layers of the arterial wall, usually due to penetrating or blunt trauma. False aneurysm of the pulmonary artery has been described in cases of Behcet's syndrome, neoplasia, pulmonary aspergillosis, septic emboli and chest trauma [3-7]. It can also be iatrogenic, especially after Swan Ganz catheter insertion and balloon inflation [2]. The aetiology in our case can simply be due to inadvertent trauma to the pulmonary artery in the first surgery. However, careful pathological examination of the excised lung from the second surgery strongly suggests infection as a precipitating factor for the development of the aneurysm in an already weakened, atherosclerotic pulmonary artery branch. Moreover, an increase in pulmonary arterial pressure post lobectomy is well documented [8], and could have had a role in the aetiology.

The gold standard for diagnosis used to be pulmonary angiography, but this is now largely superseded by noninvasive CT angiography and 3-D reconstruction. Treatment can be surgical through aneurysmectomy and/or lobectomy or radiological through steel or tungsten coil embolization. In our case, however, owing to a grossly consolidated lung and friable pulmonary vessels, pneumonectomy was elected as the safest option. 


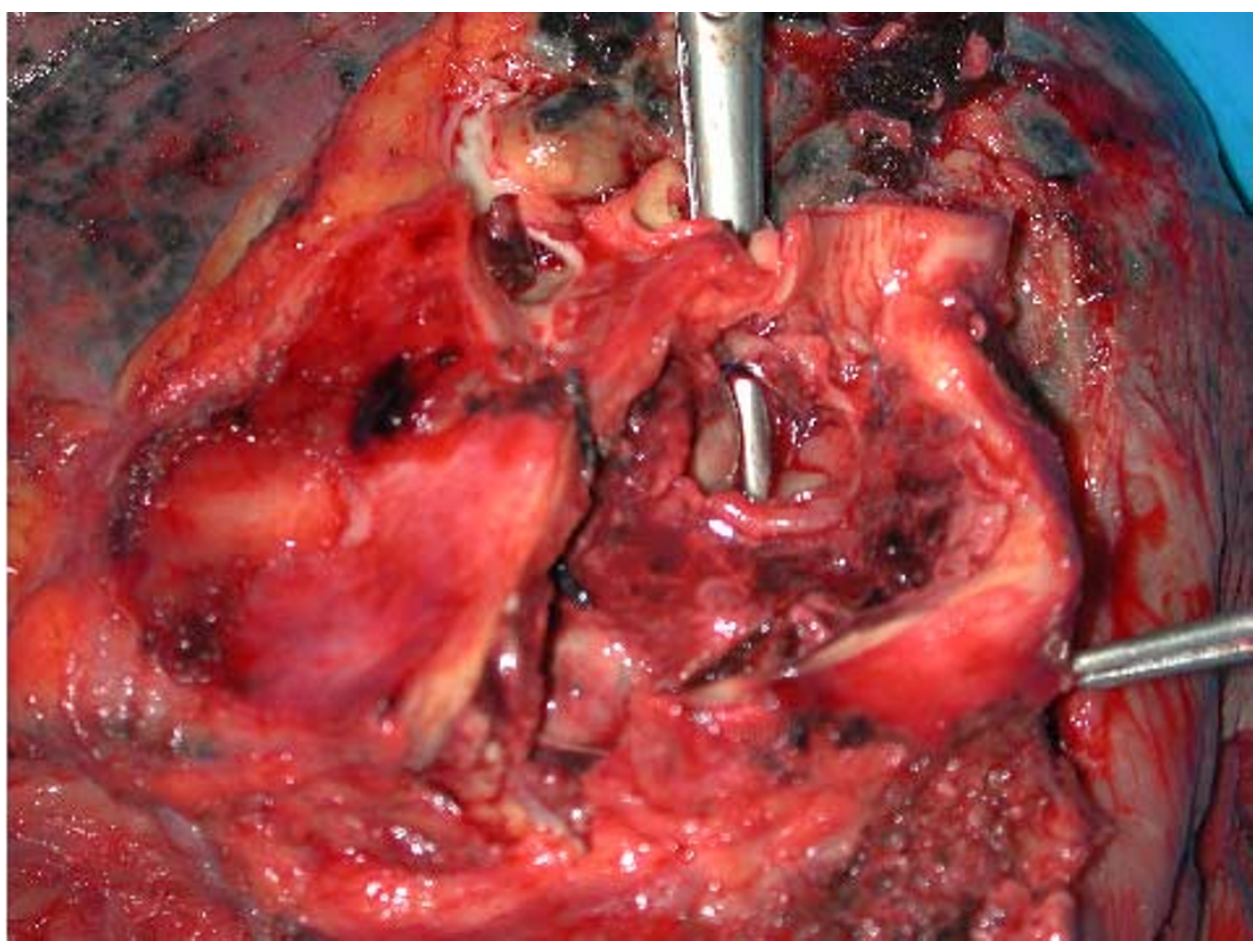

\section{Figure 2}

The false aneurysm cavity was incised and retracted open. Dissecting scissors were inserted into the bronchus intermedius. These can be clearly seen through the bronchovascular fistula.

\section{Competing interests}

The author(s) declare that they have no competing interests.

\section{Authors' contributions}

HS was involved in collection of data and material used during preparation of the paper. HS was involved in the editing and drafting of the paper. JR was involved in the primary and revision surgery and contributed to the initial draft. SC is the lead clinician involved in the care of the patient and the prime supervisor of the work. All authors read and approved the final manuscript.

\section{Acknowledgements}

Reproduction of the CT scans was carried out at Freeman Laboratories Reprographic Services, funded by Newcastle upon Tyne Hospitals NHS Trust, United Kingdom.

Authors received no external funding for the preparation of this paper. There was no external medical writer involved.

Consent was given by the patient for the case report to be published

\section{References}

I. DeLima LG, Wynands JE, Bourke ME, Walley VM: Catheterinduced pulmonary artery false aneurysm and rupture: case report and review. Journal of Cardiothoracic and Vascular Anesthesia 1994, 8:70-75.

2. Poplausky MR, Rozenblit G, Rundback JH, Crea G, Maddineni S, Leonardo R: Swan-Ganz catheter-induced pulmonary artery pseudoaneurysm formation: three case reports and a review of the literature. Chest 200I, I 20:2 I05-II.

3. Yassine N, el Meziane A, Alaoui-Yazidi A, Bartal M: Aneurysms of the pulmonary artery in Behcet disease. Apropos of 5 new cases. Rev Pneumol Clin 1997, 53:42-8.

4. Oliver TB, Stevenson AJ, Gillespie IN: Pulmonary artery pseudoaneurysm due to bronchial carcinoma. Br J Radiol 1997, 70:950-I.

5. Caulet S, Capron F, Laaban JP, Prudent J, Rochemaure J, Diebold J: Fatal hemoptysis during bronchial aspergillosis with multiple pulmonary artery aneurysms. Ann Pathol 1990, 10:177-80.

6. Ungaro R, Saab S, Almond CH, Kumar S: Solitary peripheral pulmonary artery aneurysms. Pathogenesis and surgical treatment. J Thorac Cardiovasc Surg 1976, 71:566-7I.

7. de Jonge Ingeborg, Vahl Anco, van der Hulst Victor: Coil Embolization of a Left Pulmonary Artery Pseudoaneurysm After Penetrating Injury. Journal of Endovascular Therapy I0(3):68I-683.

8. Volkner E, Bartel M, Mohorn M, Leonhardt R, Schaefer R, Rupprecht $\mathrm{H}$ : Haemodynamic and respiratory changes after lung resection. Zentralbl Chir 1977, 102:592-7. 